\title{
$\left.{ }_{\text {SCIENCE }}^{\text {MATRIX }}\right\}$ Mascot Search Results
}

\section{Protein View}

Match to: OMPA_SALTY Score: 85 Expect: 4.3e-005

Outer membrane protein A OS=Salmonella typhimurium (strain LT2 / SGSC1412 / ATCC 700720) GN=ompA PE=3 SV=2

Nominal mass $\left(\mathrm{M}_{\mathrm{r}}\right)$ : $\mathbf{3 7 4 9 2}$; Calculated $\mathrm{pI}$ value: $\mathbf{5 . 6 0}$

NCBI BLAST search of OMPA SALTY against $\mathrm{nr}$

Unformatted sequence string for pasting into other applications

Taxonomy: Salmonella enterica subsp. enterica serovar Typhimurium str. LT2

Variable modifications: Carbamidomethyl (C), Oxidation (M)

Cleavage by Trypsin: cuts C-term side of KR unless next residue is $P$

Number of mass values searched: 38

Number of mass values matched: 10

Sequence Coverage: $30 \%$

Matched peptides shown in Bold Red

1 MKKTAIAIAV ALAGFATVAQ AAPKDNTWYA GAKLGWSQYH DTGFIHNDGP

51 THENQLGAGA FGGYQVNPYV GFEMGYDWLG RMPYKGDNIN GAYKAQGVQI

101 TAKLGYPITD DLDVYTRLGG MVWRADTKSN VPGGPSTKDH DTGVSPVFAG

151 GIEYAITPEI ATRLEYQWTN NIGDANTIGT RPDNGLLSVG VSYRFGQQEA

201 APVVAPAPAP APEVQTKHFT LKSDVLFNFN KSTLKPEGQQ ALDQLYSQLS

251 NLDPKDGSVV VLGFTDRIGS DAYNQGLSEK RAQSVVDYLI SKGIPSDKIS

301 ARGMGESNPV TGNTCDNVKP RAALIDCLAP DRRVEIEVKG VKDVVTQPOA

351

Show predicted peptides also

Sort Peptides By $\odot$ Residue Number $\bigcirc$ Increasing Mass $\bigcirc$ Decreasing Mass

$\begin{array}{rrrr}\text { Start }- \text { End } & \text { Observed } & \text { Mr (expt) } & \text { Mr (calc) } \\ 25-33 & 1025.5163 & 1024.5091 & 1024.4614 \\ 104-117 & 1640.7939 & 1639.7866 & 1639.8094 \\ 223-231 & 1083.5850 & 1082.5778 & 1082.5397 \\ 256-267 & 1264.6474 & 1263.6401 & 1263.6460 \\ 268-280 & 1381.6572 & 1380.6499 & 1380.6521 \\ 268-281 & 1537.7272 & 1536.7200 & 1536.7532 \\ 281-292 & 1378.7417 & 1377.7344 & 1377.7616 \\ 303-321 & 2032.9803 & 2031.9731 & 2031.9102 \\ 322-332 & 1214.6249 & 1213.6176 & 1213.6125 \\ 333-339 & 872.5051 & 871.4978 & 871.5127\end{array}$

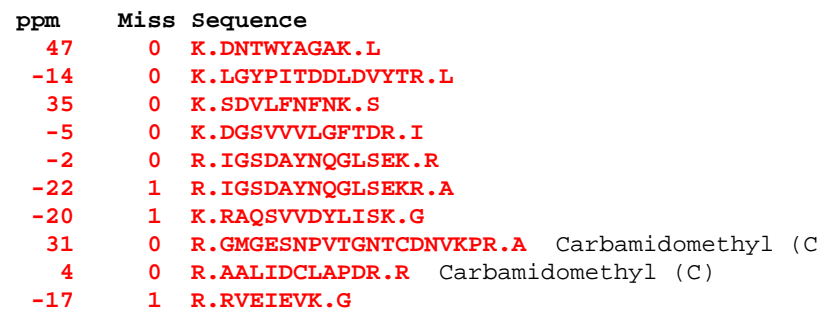

No match to: $644.1343,662.4507,818.5446,832.3867,833.0637,842.6634,855.0318,870.6121,870.9993,1059.9536,1106.6079$
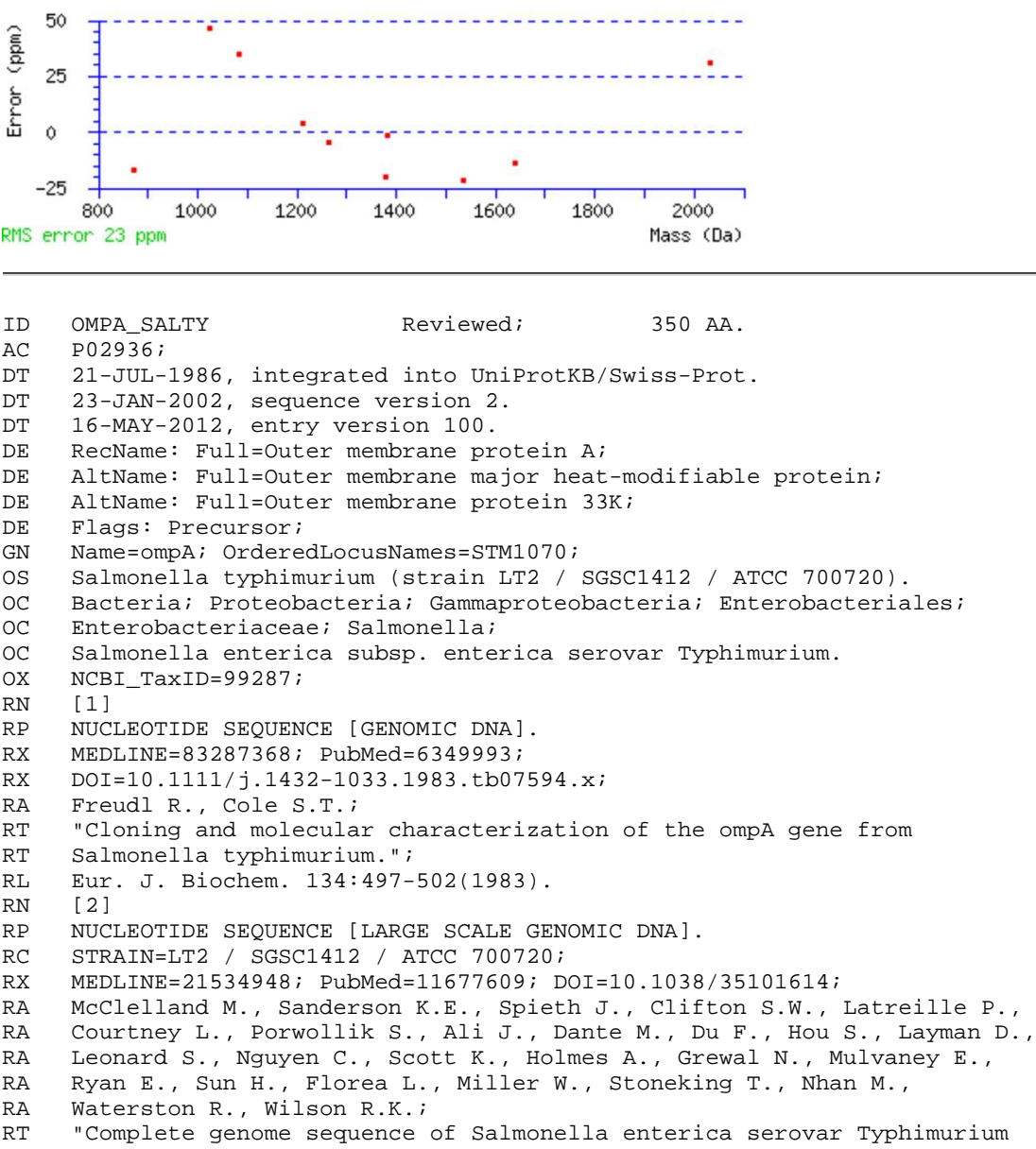

350 AA.

21-JUL-1986, integrated into UniProtKB/Swiss-Prot.

23-JAN-2002, sequence version 2.

16-MAY-2012, entry version 100.

RecName: Full=Outer membrane protein $A$

AltName: Full=Outer membrane major heat-modifiable protein;

AltName: Full=Outer membrane protein $33 \mathrm{~K}$;

Flags: Precursor;

Name $=O \mathrm{mPA} ;$ OrderedLocusNames $=\mathrm{STM1070}$;

Salmonella typhimurium (strain LT2 / SGSC1412 / ATCC 700720).

Bacteria; Proteobacteria; Gammaproteobacteria; Enterobacteriales;

Enterobacteriaceae; Salmonella;

Salmonella enterica subsp. enterica serovar Typhimurium.

NCBI_TaxID $=99287$

[1]

NUCLEOTIDE SEQUENCE [GENOMIC DNA].

MEDLINE=83287368; PubMed=6349993;

$\mathrm{DOI}=10.1111 / j .1432-1033.1983 . t b 07594 . x$

Freudl R., Cole S.T.;

"Cloning and molecular characterization of the ompA gene from

Salmonella typhimurium."

Eur. J. Biochem. 134:497-502(1983).

[2]

NUCLEOTIDE SEQUENCE [LARGE SCALE GENOMIC DNA].

STRAIN=LT2 / SGSC1412 / ATCC 700720;

MEDLINE $=21534948 ;$ PubMed=11677609; DOI=10.1038/35101614;

McClelland M., Sanderson K.E., Spieth J., Clifton S.W., Latreille P.,

Courtney L., Porwollik S., Ali J., Dante M., Du F., Hou S., Layman D.,

Leonard S., Nguyen C., Scott K., Holmes A., Grewal N., Mulvaney E.,

Ryan E., Sun H., Florea L., Miller W., Stoneking T., Nhan M.,

Waterston R., Wilson R.K.

"Complete genome sequence of Salmonella enterica serovar Typhimurium 


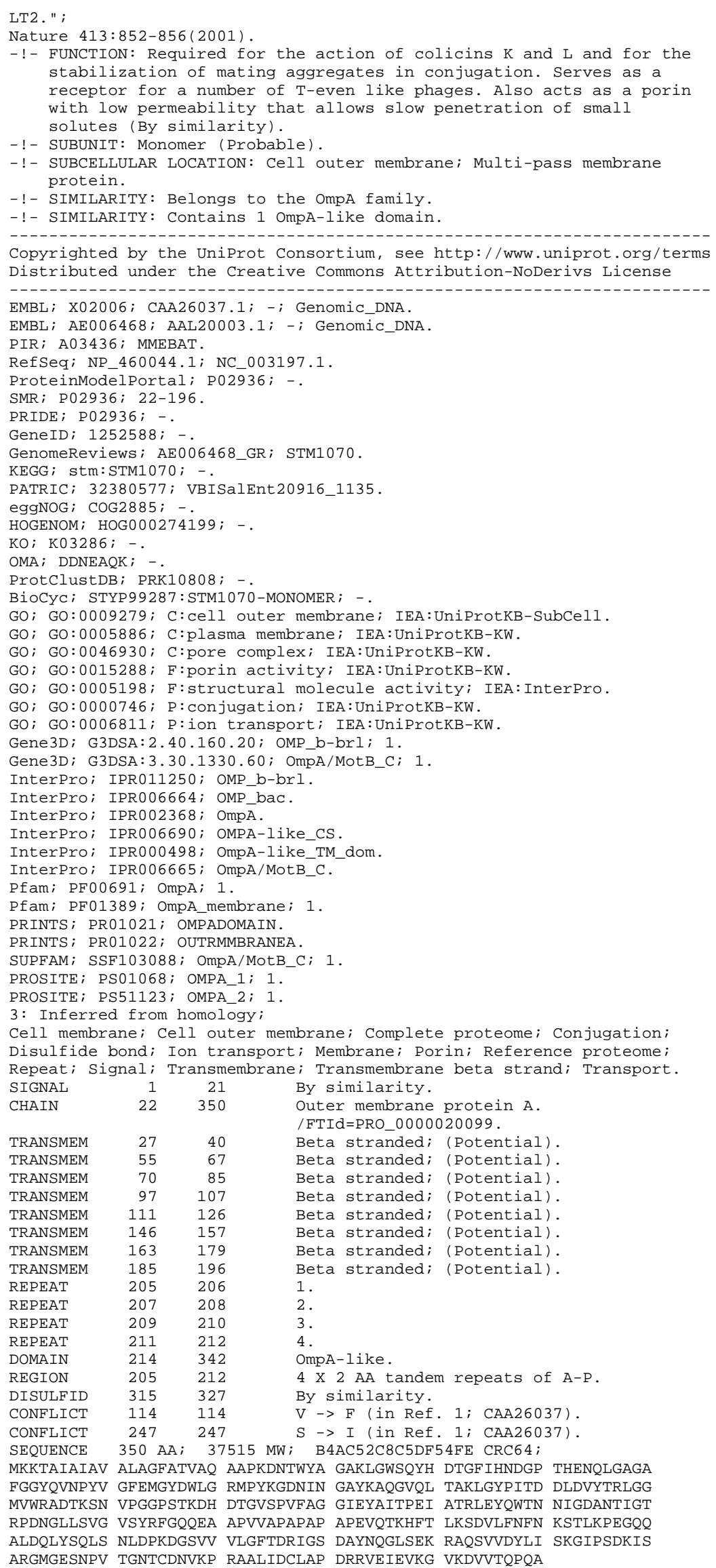
MVWRADTKSN VPGGPSTKDH DTGVSPVFAG GIEYAITPEI ATRLEYQWTN NIGDANTIGT RPDNGLLSVG VSYRFGQQEA APVVAPAPAP APEVQTKHFT LKSDVLFNFN KSTLKPEGQQ ALDQLYSQLS NLDPKDGSVV VLGFTDRIGS DAYNQGLSEK RAQSVVDYLI SKGIPSDKIS ARGMGESNPV TGNTCDNVKP RAALIDCLAP DRRVEIEVKG VKDVVTQPOA 